\title{
Transmission of 61 C-band Channels over Record Distance of Hollow-Core-Fiber with L-band Interferers
}

\author{
Antonino Nespola, Stefano Straullu, Thomas D. Bradley, Kerrianne Harrington, Hesham Sakr, Gregory T. Jasion, \\ Eric Numkam Fokoua, Yongmin Jung, Yong Chen, John R. Hayes, Fabrizio Forghieri, David J. Richardson, \\ Francesco Poletti, Gabriella Bosco, Pierluigi Poggiolini
}

\begin{abstract}
We report on two recirculating loop transmission experiments over a hollow-core fiber of the Nested-Antiresonant Nodeless type (NANF). We transmitted 61 channels in C-band at 32 GBaud, with either PM-QPSK or PM-16QAM modulation. In addition, $61 \mathrm{~L}$-band interferers co-propagated in the NANF at all times, though they were not recirculated in the loop, to check for the presence of possible crosstalk effects between $C$ and $L$-band in the NANF. The loop comprised the longest NANF transmission line yet constructed $(7.72 \mathrm{~km})$, as well as $55 \mathrm{~km}$ of pure-silicacore fiber (PSCF) needed to provide enough signal buffering and EDFA stabilization for the loop. The launch power into the PSCF was low enough to avoid generation of any significant non-linear noise.

Using PM-QPSK, we achieved a record $618 \mathrm{~km}$ transmission in NANF (80 recirculations), at an overall average GMI of 3.44 bits/symb. Using PM-16QAM, we achieved a record $201 \mathrm{~km}$ transmission, at an overall average GMI of $7 \mathrm{bits} / \mathrm{symb}$. We saw no adverse effect from the presence of $L$-band interferers in the NANF.

If progress in the reduction of NANF loss and inter-modal interference continues at the rate of the last few years, these hollow-core fibers might become a promising alternative in the quest for next-generation higher-throughput fibers, given their theoretical potential of achieving low loss and ultra-low nonlinearity over ultra-wide bandwidths, ideally bringing about a many-fold increase in throughput per fiber.
\end{abstract}

Index Terms-hollow-core fiber, NANF, coherent systems, longhaul transmission

\section{INTRODUCTION}

$\mathbf{L}$ IGHT transmission in a Hollow-Core Fiber (HCF) promises many advantages over transmission through

Antonino Nespola and Stefano Straullu are with Links Foundation, 10129 Torino, Italy (e-mail: antonino.nespola@linksfoundation.com; stefano.straullu@linksfoundation.com).

Thomas D. Bradley, Kerrianne Harrington, Hesham Sakr, Gregory T. Jasion, Eric Numkam Fokoua, Yongmin Jung, Yong Chen, John R. Hayes, David J. Richardson and Francesco Poletti are with the Optoelectronics Research Centre, University of Southampton, SO17 1BJ, UK.

Fabrizio Forghieri is with CISCO Photonics, 20871 Vimercate (MB), Italy (e-mail: fforghie@cisco.com).

Gabriella Bosco and Pierluigi Poggiolini are with Politecnico di Torino, 10129 Torino, Italy (e-mail: gabriella.bosco@polito.it; pierluigi.poggiolini@polito.it).

This research was supported by the PhotoNext initiative of Politecnico di Torino, by the CISCO SRA OPTSYS-2020, by the European Research Council (ERC) grant agreement n. 682724, by the EPSRC Airguide Photonics (EP/P030181/1), by Lumenisity and by the UK Royal Academy of Engineering. We also thank Lumentum Italy for supplying the dual-polarization MZ modulator.

Manuscript received June $30^{\text {th }}, 2020$. conventional glass-core fiber: lower latency, much lower nonlinearity, less backscattering and less sensitivity to perturbations [1], [3].

Amongst the numerous potential applications, ultrabroadband data transmission is arguably one of the most ambitious and potentially impactful ones for the technology. Early data transmission experiments performed on very shortreach links (100-300 meters) of Photonic Band-Gap HCFs (or PBGFs), progressed from single channel $10 \mathrm{Gbit} / \mathrm{s}$ OOK [2] to $40 \mathrm{Gbit} / \mathrm{s}$ WDM [3], up to high data throughput (76 Tbit/s) exploiting 256-QAM with polarization and mode multiplexing [4]. Meanwhile, other studies assessed the transmission potential of PBGFs over longer distances: $2.75 \mathrm{~km}$ using a commercial 100G interface [5] and a single channel $20 \mathrm{Gbit} / \mathrm{s}$ QPSK transmission experiment over $11 \mathrm{~km}$ with $66 \mathrm{~dB}$ loss [6].

However, when PBGFs were first inserted in a recirculating loop to test their transmission performance over even longer distances, both their fairly large loss and intermodal cross-talk (or Inter-Modal Interference, IMI) appeared as rather limiting. The best achieved result was transmission of a single PMQPSK channel, with two untested side channels added for spectral equalization, recirculated through a single span of $6.2 \mathrm{~km}$ of PBGF with $48.1 \mathrm{~dB}$ loss [7]. Assuming a pre-FEC BER limit of $5 \cdot 10^{-2}$, a maximum transmission length through PBGF of $55.8 \mathrm{~km}$ and $74.8 \mathrm{~km}$ was achieved at 32 GBaud and 14 GBaud, respectively.

A considerable improvement in performance was obtained by switching to a different type of HCF, the Nested Antiresonant Nodeless Fiber (NANF) [8]. The NANF not only enables considerably lower loss (current record: $0.28 \mathrm{~dB} / \mathrm{km}$ [13]) and broader potential optical transmission bandwidths (several hundreds of nanometers [10]) than PBGFs, but thanks to an inherent and distributed high-order mode stripping mechanism, NANFs can also considerably reduce the IMI that impairs long distance data transmission. The current record transmission length through NANF is $341 \mathrm{~km}$, achieved by recirculating 71 times a PM-QPSK channel, operating at 32 GBaud, through a $4.8 \mathrm{~km}$ assembled line of NANF, with $7.5 \mathrm{~dB}$ loss. The channel sat at the center of a 61-channel WDM comb in the $\mathrm{C}$-band and the record length was achieved at a pre-FEC BER of less than $3 \cdot 10^{-2}$ [11]. This result improved considerably over [7]. However, as in [7], only one channel was measured: WDM transmission was not tested. 

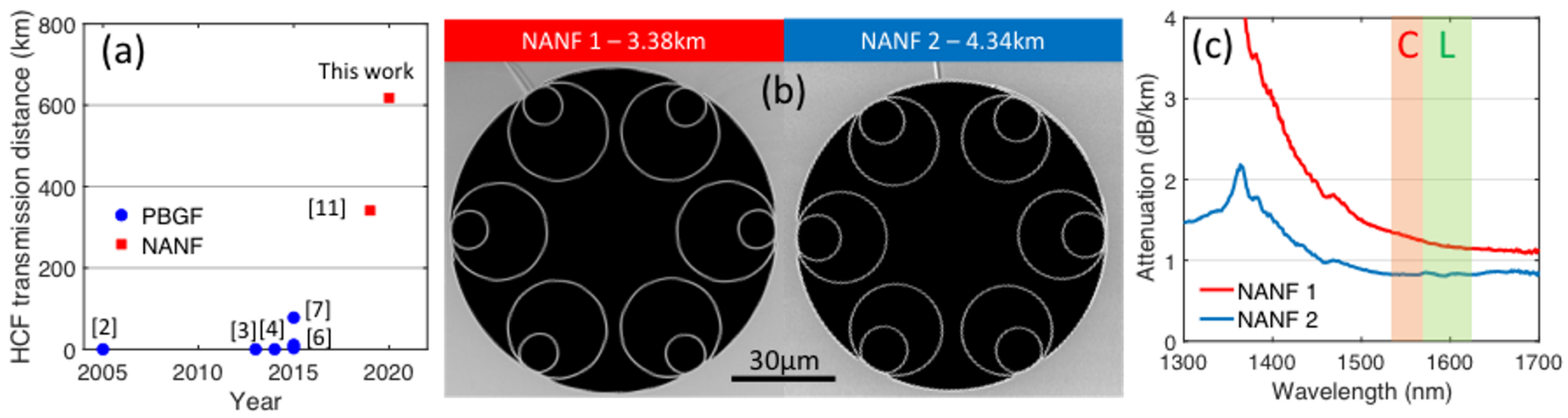

Fig. 1. (a) Evolution of data transmission distance through HCFs; (b) cross sectional SEMs of the two fibers; (c) Cutback loss.

In this work, we managed to produce and assemble the longest NANF transmission line yet constructed $(7.72 \mathrm{~km})$, obtained by splicing the longest continuous length of NANF so far reported, $4.34 \mathrm{~km}$, to another $3.38 \mathrm{~km}$ long stretch of NANF. The average estimated NANF loss at $1550 \mathrm{~nm}$ through the whole line was $0.84 \mathrm{~dB} / \mathrm{km}$. The total insertion loss of the assembled NANF line, including NANF/NANF and NANF/SMF splices, was $9.1 \mathrm{~dB}$. The NANF transmission line was placed into a recirculating loop that carried one 32-GBaud PM-QPSK modulated channel, as well as spectrally-shaped ASE to emulate another 60 interfering WDM channels in Cband. The modulated channel could be tuned across the whole comb, so that transmission could be tested for all channels.

The NANF was also loaded with spectrally-shaped ASE to emulate further 61 interfering WDM channels in the L-band. These L-band interferers co-propagated in the NANF at all times, though they were not recirculated in the loop, to check for the possible presence of crosstalk effects between $\mathrm{C}$ and L-band in the NANF.

Note that the loop also included $55 \mathrm{~km}$ of pure silica-core fiber (PSCF), whose purpose was that of providing sufficient signal loading capability and a stabilizing effect for the loop EDFAs. The launch power into the PSCF was low enough to avoid generation of any significant non-linear noise.

Setting a threshold GMI of 3.55 bits/symbol, the center channel reached $772 \mathrm{~km}$ (100 recirculations), more than twice the previous directly comparable record [11]. We also achieved 80 recirculations $(618 \mathrm{~km})$ at a GMI average across all WDM channels of 3.44 bits/symb. This is the current WDM record length for coherent transmission over any HCF.

We then proceeded to test the same set-up with a higherorder constellation, PM-16QAM. In [11] a similar experiment had been carried out, reaching $125 \mathrm{~km}$, but only $1 / 4$ of the channels were tested. In this experiment, we reached $201 \mathrm{~km}$ at an average GMI of 7 bits/symb, measured across all WDM channels, setting the current transmission length record for PM-16QAM over HCF.

We saw no performance degradation on either experiment when launching the L-band interferers into the NANF, together with the C-band WDM comb. Even though this was the expected outcome due to the theoretically very low nonlinearity of the NANF, this is nonetheless, we believe, the first time this has ever been experimentally tested.
These record results greatly improve over [11]. A picture of reported record transmission distances over any HCFs is provided in Fig. 1(a), for comparison. The steep rise shown in the figure is due to the very significant progress that has been made lately in NANF design, fabrication and overall performance. Over the course of a few years, NANF loss has fallen from $10 \mathrm{~dB} / \mathrm{km}$ in 2017 to the current record-low value of $0.28 \mathrm{~dB} / \mathrm{km}$ [13], close to silica-core fiber values. While some challenges still need to be addressed, this bodes well for the possibility of NANFs becoming a practical option for high-capacity optical systems in the not-too-distant future.

This paper is organized as follows. In Sect. II the NANF spools and overall assembled NANF line are described in detail and their performance is discussed. In Sect. III the experimental system set-up is introduced. In Sects. IV and $\mathrm{V}$, the PM-QPSK and PM-16QAM transmission results are shown and discussed. Sect. VI is devoted to discussing the Lband interference test and results. Comments and conclusion follow.

This paper is a follow-up to the OFC 2020 post-deadline paper [14]. Here, substantially more details have been provided on the PM-QPSK experiment. Also, the PM-16QAM experiment has been added, which was not present in [14].

\section{ASSEMBLY OF A 7.72 KM NANF SPAN}

NANFs from two separate draws and different preforms, both designed to operate in the first antiresonant window, were spliced together to form a $7.72 \mathrm{~km}$ assembled line. Their scanning electron micrographs (SEMs) are shown in Fig. 1(b). The two fibers are geometrically similar, with a difference in the size of their inner tubes.

NANF $1(3.38 \mathrm{~km})$, also employed in the previous experiment [11], has an average core diameter and membrane thickness of $35.6 \mu \mathrm{m}, 0.55 \mu \mathrm{m}$, respectively. Its loss (Fig. 1(c)) decreases from $1.37 \mathrm{~dB} / \mathrm{km}$ at $1530 \mathrm{~nm}$ to $1.15 \mathrm{~dB} / \mathrm{km}$ at $1625 \mathrm{~nm}(1.30 \mathrm{~dB} / \mathrm{km}$ at $1550 \mathrm{~nm})$.

NANF 2 was fabricated with a similar core diameter and membrane thickness $(35.9 \mu \mathrm{m}, 0.48 \mu \mathrm{m})$. However, by finessing the fabrication procedures, we managed to make NANF 2 the longest NANF from a single draw to date $(4.34 \mathrm{~km})$. In addition, NANF 2 has a considerably lower loss $(0.82 \mathrm{~dB} / \mathrm{km}$, Fig. 1(c)) than NANF 1, spectrally flat from 1530 to $1625 \mathrm{~nm}$, 


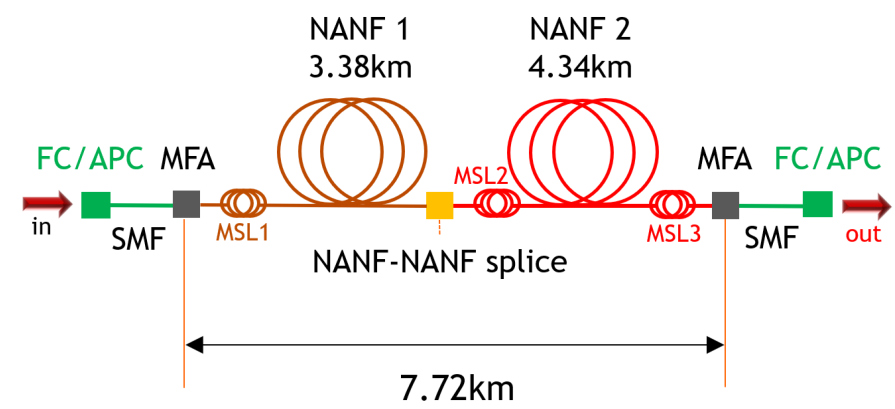

Fig. 2. Schematic of the NANF line used in the experiment. SMF: standard single-mode fiber. MFA: mode-field adapter. FC/APC: fiber connector with angled physical contact. MSL: mode stripping loops.

not far from the value of $0.65 \mathrm{~dB} / \mathrm{km}$ which was the recordlow NANF loss [9] when the experiments of this paper were carried out (only recently surpassed by [13]).

The overall assembled NANF line used in the experiment is shown in Fig. 2. The fibres were spooled on standard $1 \mathrm{~m}$ circumference bobbins. Its total measured loss was $8.7 \mathrm{~dB}$. Besides the NANFs, loss was due to splicing to SMF patchcords using mode field adapters (MFAs, $1 \mathrm{~dB}$ loss per end) and to the mid-span NANF-NANF splice $(0.2 \mathrm{~dB})$. Interestingly, taking out splice loss, the estimated pure spooled NANF propagation loss was lower than suggested by the cutback measurements: $6.50 \mathrm{~dB}$ total or $0.84 \mathrm{~dB} / \mathrm{km}$ average. The discrepancy is potentially caused by the fact that the loss curves of both NANFs shown in Fig.1(c) were measured by cutting back the fibers to only $10 \mathrm{~m}$. This can lead to an overestimated loss reading when the high order mode content in the fiber is not fully suppressed in the short fiber length.

Once the experiment got underway, it was found that substantial IMI was present in the line. To mitigate it, modestripping fiber loops were created along the line. Because of this, the actual overall loss of the NANF line went up to $9.1 \mathrm{~dB}$, total. For a detailed description and discussion of this aspect, see Sect. IV

\section{EXPERIMENT SET-UP}

A total of 122 WDM channels (61 in the C-band and 61 in the L-band) were emulated by shaping ASE noise as raised-cosine spectra with $32 \mathrm{GHz}$ bandwidth, roll-off factor 0.2 and $50 \mathrm{GHz}$ spacing. The overall measured transmitted signal spectrum is shown in Fig. 3(a). The shaping was done using high-resolution programmable optical filters (Finisar Waveshapers) as shown in Fig. 3(b), where only the C-band setup is reported. A similar setup was used for generating the L-band WDM comb, but without the modulated channel.

For transmission performance measurements, in turn each one of the 61 ASE-emulated C-band channels was turned off and replaced by an actual modulated Channel Under Test (CUT). The C-band transmitter schematic is shown in Fig. 3(b). The CUT used an External Cavity Laser (ECL) with linewidth less than $100 \mathrm{kHz}$, which was modulated either with PM-QPSK or PM-16QAM at 32 GBaud by means of a dualpolarization Mach-Zehnder Modulator (MZM), driven by four

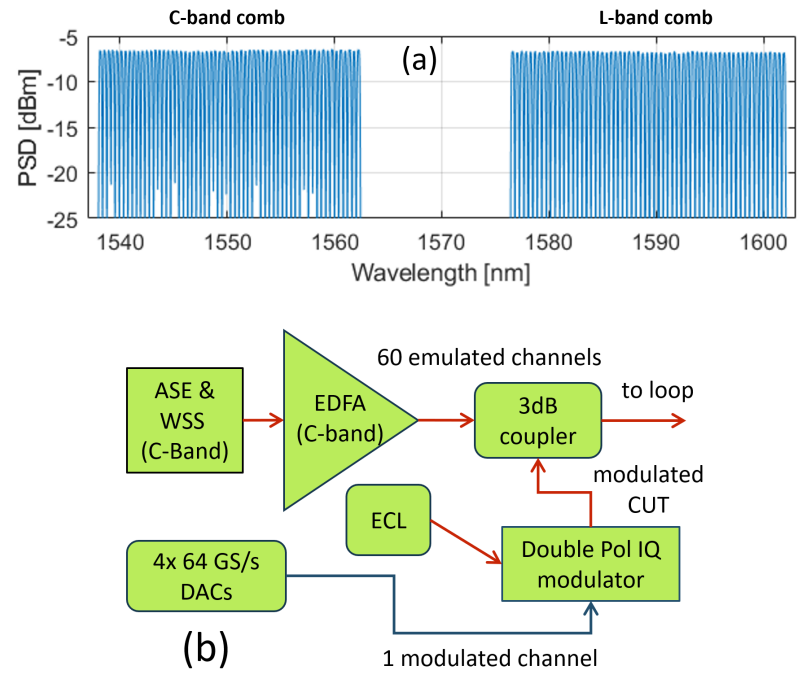

Fig. 3. (a): power spectral density (PSD) of the $(\mathrm{C}+\mathrm{L})$-band 122-channel WDM spectrum. (b): C-band transmitter schematic.

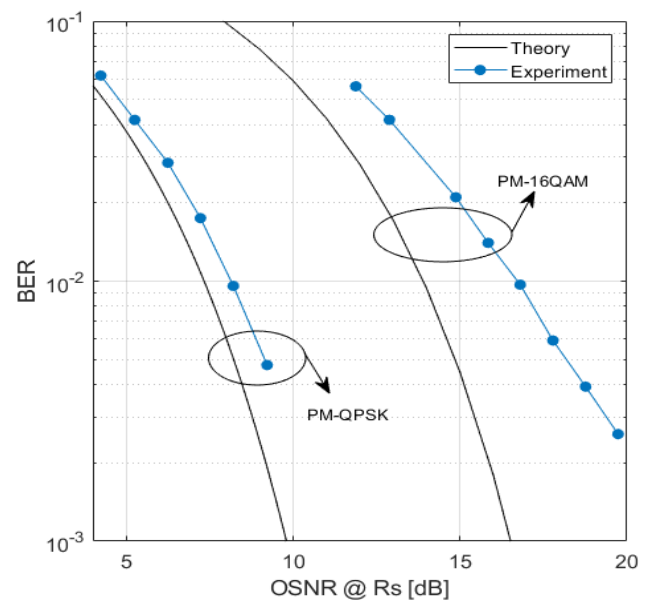

Fig. 4. Back-to-back BER vs. OSNR of both PM-QPSK and PM-16QAM Tx/Rx pairs. Solid black lines: ideal curves in AWGN. Blue circles: measured values.

$64 \mathrm{GS} / \mathrm{s}$ DACs. The CUT spectrum was raised-cosine with 20\%-roll-off. The back-to-back performance of the CUT is shown in Fig. 4. At a pre-FEC BER of $3 \cdot 10^{-2}$ the back-toback penalty vs. ideal transceiver performance (in AWGN) was $0.65 \mathrm{~dB}$ for PM-QPSK and $2 \mathrm{~dB}$ for PM-16QAM.

The loop schematic is shown in Fig. 5. The loop recirculated the C-band WDM comb, while the L-band comb went through the NANF without recirculating, as explained below. The loop consisted of four sections, each starting with an EDFA. EDFA 1 launched the C-band WDM comb at $19 \mathrm{dBm}$ into the $7.72 \mathrm{~km}$ NANF line. It was followed by EDFA 2 feeding a high-resolution programmable optical filter (Finisar Waveshaper) filter tasked with flattening the overall loop transfer function, followed by EDFA 3 and a spool of $55 \mathrm{~km}$ of pure silica-core fiber (PSCF) with $150 \mu \mathrm{m}^{2}$ effective area and attenuation of $0.165 \mathrm{~dB} / \mathrm{km}$, followed by EDFA 4 feeding an acousto-optic modulator, a $2 \times 2$ splitter/combiner 


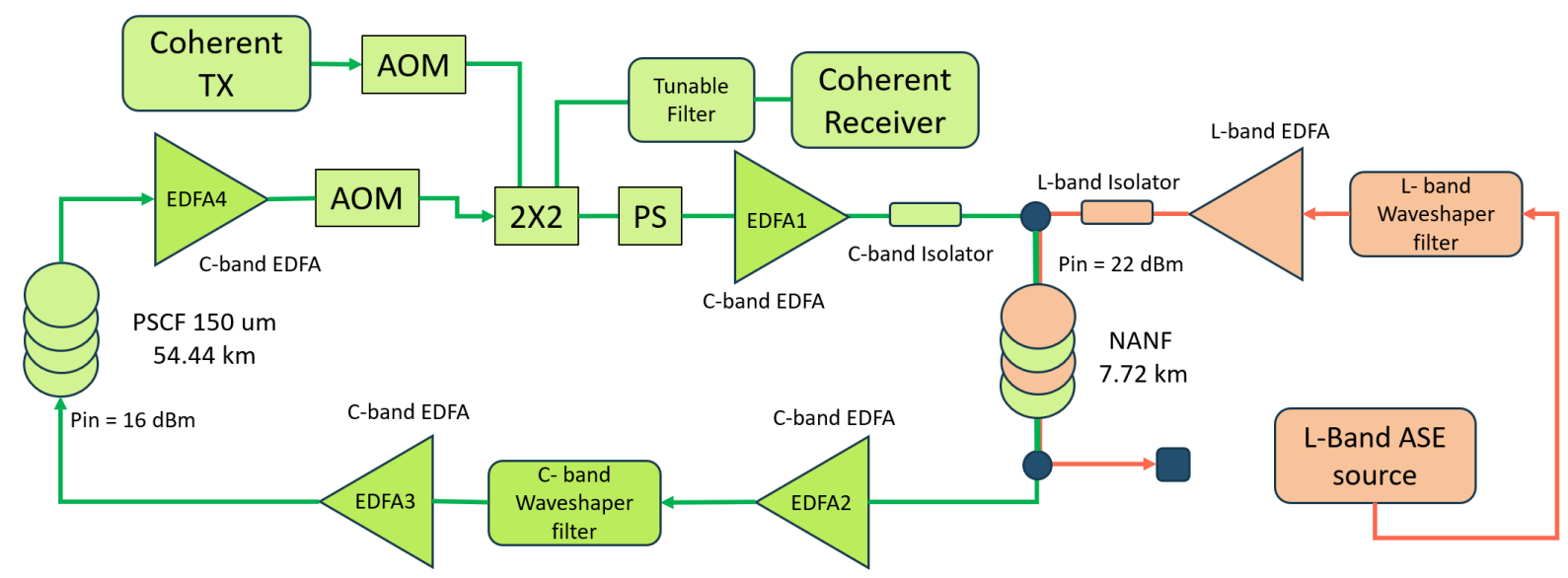

Fig. 5. Loop schematic. Green arcs: C-band signal. Pink arcs: L-band signal.

and a synchronous polarization scrambler (PS), the latter used to randomize the state of polarization at each recirculation.

Note that the transmitted C-band spectrum was about $3 \mathrm{THz}$ wide, somewhat less than full C-band. The reason why the edges of the C-band were clipped is because of limited optical amplifier bandwidth. This limitation could be compensated for by the programmable optical Waveshaper filter in the loop but, being a passive component, this meant that to achieve spectral flattening it had to apply substantial extra attenuation to the rest of the WDM comb, which in turn caused substantial overall penalty. So, we decided to remove a few channels at the edges of the C-band and restrict the transmitted WDM C-band spectrum to $3 \mathrm{THz}$.

The PSCF was necessary to provide sufficient signal loading capability and long-enough round-trip delay for loop management and EDFA gain transient dampening. The EDFA 3 launch power into the PSCF was set to $16 \mathrm{dBm}$, a value which kept the amount of non-linearity noise produced in the PSCF at a negligible level as compared to other sources of signal degradation (see Sect. VII-A for more details). At this launch power, the PSCF could be effectively considered to operate in linearity.

Right before the NANF, a WDM combiner added the L-band WDM comb into the NANF. The L-band comb was launched into the NANF at the same power level as the C-band comb, as shown in Fig. 3(a), to test for possible cross-band interactions. Immediately after the NANF, a WDM splitter stripped the Lband WDM comb out of the loop. The L-band comb was not recirculated but it co-propagated in the NANF with the C-band comb.

At the receiver, a tunable optical filter selected the CUT, which was then combined with an ECL local-oscillator, with linewidth less than $100 \mathrm{kHz}$, in an integrated coherent receiver. The four electrical outputs were sampled at $50 \mathrm{Gsamp} / \mathrm{symb}$ and off-line processed. The DSP performed upsampling to 2 samp/symb, chromatic dispersion compensation and frequency offset removal. Next, the signal went through a complex 2-by-2 LMS adaptive equalizer. In the case of PM-QPSK modulation, the LMS stage was followed by a V\&V Carrier-Phase Estimation (CPE) stage. In the case of PM-16QAM modulation, a blind-phase search algorithm was used. In both cases 5\% of symbols were considered as pilot symbols and used to perform phase unwrapping and improve phase-recovery.

\section{PM-QPSK EXPERIMENT}

We first set the transmission format to PM-QPSK. Initial transmission attempts reached relatively modest maximum reach results, hinting at the presence of substantial IMI along the line.

Theoretical analyses and separate characterization indicated that the smaller tubes in NANF $1(9.88 \mu \mathrm{m})$ should produce noticeably less IMI than NANF $2(13.27 \mu \mathrm{m})$. IMI in the overall line would then mostly be caused by NANF 2 . To mitigate it, we introduced higher-order mode stripping loops. A set of loops with 5 turns of $7.3 \mathrm{~cm}$ bend diameter was wound on NANF 1 at the launch end, after the splice with the input SMF patchcord (MSL 1 in Fig. 2). Another set of loops with 5 turns of $4.8 \mathrm{~cm}$ bend diameter was wound on NANF 2 immediately after the splice with NANF 1 (MSL 2 in Fig. 2). A third set of loops with 5 turns of $4.8 \mathrm{~cm}$ bend diameter was wound on NANF 2 immediately before the splice with the output SMF patchcord (MSL 3 in Fig. 2). The three loops added $0.4 \mathrm{~dB}$ loss to the line, raising it from 8.7 to $9.1 \mathrm{~dB}$, but considerably reducing IMI and improving transmission performance. Note that the loop diameter and the number of loops were chosen empirically as an acceptable trade-off between introducing as little additional loss on the fundamental mode and as large an attenuation on the higherorder modes as possible. For more details on multimode propagation in NANFs and on the possible generation of IMI, please see [8].

Fig. 6 shows the GMI-vs.-recirculations for the PM-QPSK center channel (number 31 out of 61) of the C-band comb. Assuming a threshold of $3.55 \mathrm{bits} / \mathrm{symb}$ (equivalent to a preFEC BER in AWGN of $3 \cdot 10^{-2}$ ) its max-reach was 100 recirculations, corresponding to about $772 \mathrm{~km}$ in NANF. A similar measurement was done in [11], where the center channel reached $341 \mathrm{~km}$, representing the previous record transmission distance in NANF.

The loop was then set to 80 recirculations for the full WDM comb measurement, or $618 \mathrm{~km}$ in NANF. Dots in Fig. 7(b) 


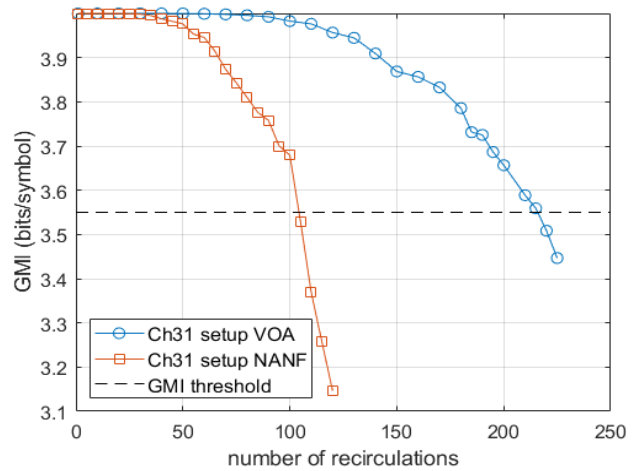

Fig. 6. PM-QPSK experiment, GMI vs. recirculations for the C-band center channel, with VOA in the loop (circles) or NANF in the loop (squares).
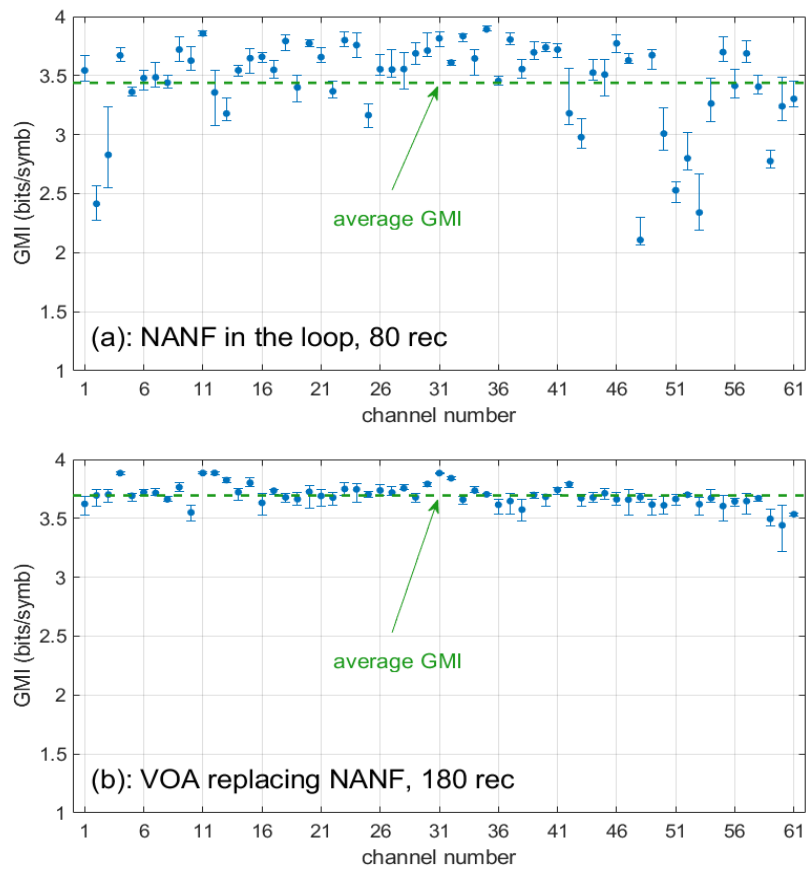

Fig. 7. PM-QPSK experiment. Total GMI (both polarizations) for all C-band channels. Six measurements per channel. Dots indicate the mean value, bars go from lowest to highest value. Higher channel index means higher channel frequency, from 191.9 THz (channel \# 1) to $194.9 \mathrm{THz}$ (channel \# 61). (a) NANF in the loop, 80 recirculations (618 $\mathrm{km}$ in NANF). (b): VOA in the loop with same attenuation as NANF, 180 recirculations.

shows the GMI (sum of both polarizations) of each C-band channel, averaged over 6 measurements. Vertical bars range between the min and max measured value for each channel. The mean GMI across all channels was 3.44 bits/symb.

A distinctive feature of the GMI plot was however the non-uniformity of the results. Some channels operated at or above 3.8 bits/symb, whereas others were recorded well below 3 bits/symb. In addition, theoretical calculations based exclusively on loop ASE noise accumulation predicted a substantially longer reach. Non-uniformity and shorter reach than expected suggest that the NANF still caused non-negligible IMI, even with the mode-stripping loops in the line.

To investigate this aspect, we replaced the NANF in the loop with a variable optical attenuator (VOA), set to exactly match
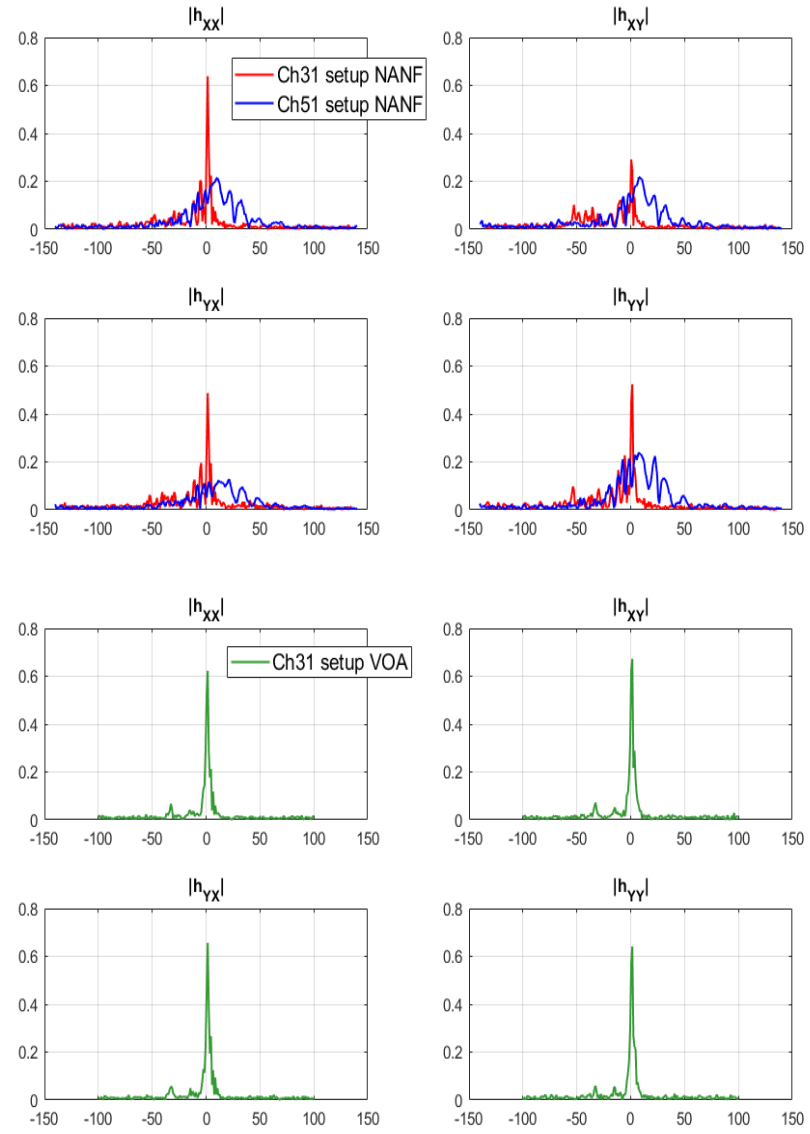

Fig. 8. Absolute value of the impulse responses of the LMS equalizer filters at 80 recirculations. Abscissa: sample number. Ordinate: arbitrary units. Top 4 plots: with the NANF in the loop, totaling $618 \mathrm{~km}$ through NANF. Red lines: channel \#31 (average GMI 3.81 bits/symb). Blue lines: channel \#51 (average GMI 2.53 bits/symb). Bottom 4 plots: with the VOA in the loop. All sets of responses normalized so that their $h_{x x}$ component has unit energy.

the NANF attenuation. Fig. 6 shows the GMI vs. recirculations for the center channel in this condition. Transmission reached 215 recirculations at a GMI of 3.55 bits/symb, more than twice the recirculations was reached by the center channel with the NANF. We also tested the whole WDM comb at 180 recirculations with VOA replacing the NANF, Fig. 7(b). The figure shows a total mean GMI across all channels of 3.7 bits/symb at 180 recirculations, vs. 3.44 with the NANF at 80 recirculation. Uniformity appears to be much better than in Fig. 7(a). These results clearly indicate that substantial signal degradation was occurring in the NANF.

A strong hint that IMI could be responsible for such degradation comes from the plot of the LMS equalizer filters impulse responses (absolute values), shown in Fig. 8, at 80 recirculations. The top four plots show the filters for the center channel (\#31, blue lines), whose average GMI was relatively high (3.81 bits/symb), and for channel \#51 (red lines), whose average GMI was low (2.53 bits/symb). The bottom four plots show the filters for the center channel when a VOA replaced the NANF (GMI $\approx 4$ bits/symb). For each set the $h_{x x}$ response was normalized to have unit energy. The normalizing factor found for $h_{x x}$ was then used to normalize the other 

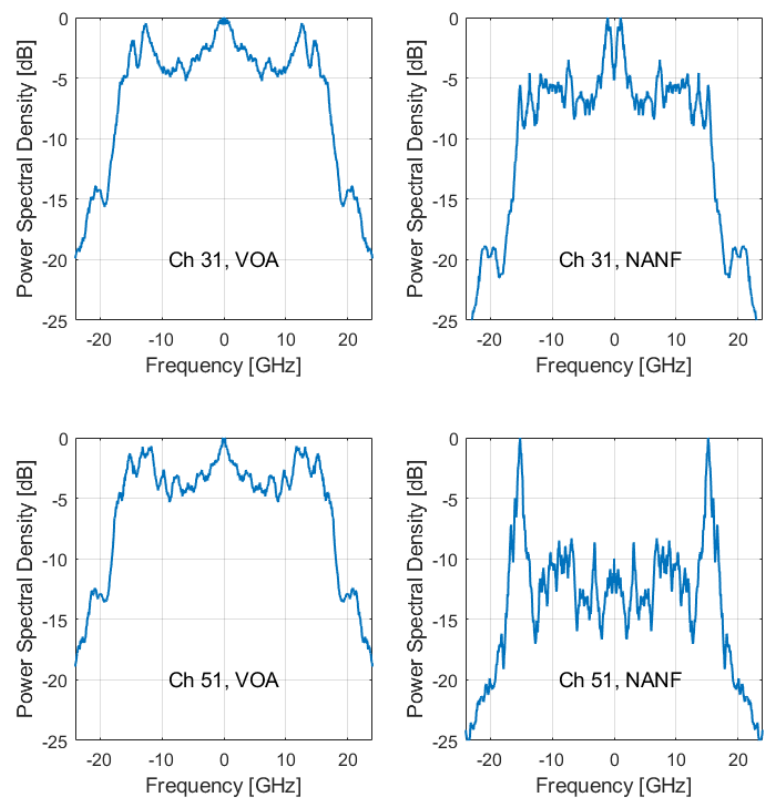

Fig. 9. Signal power spectral density at the receiver, at 80 recirculations. Left plots: when a variable-optical attenuator (VOA) is in the loop. Right plots: when the NANF is in the loop, totaling $618 \mathrm{~km}$ through NANF. Top plots: the center C-band channel, \#31 (average GMI 3.81 bits/symb). Bottom plots: channel \#51 (average GMI 2.53 bits/symb).

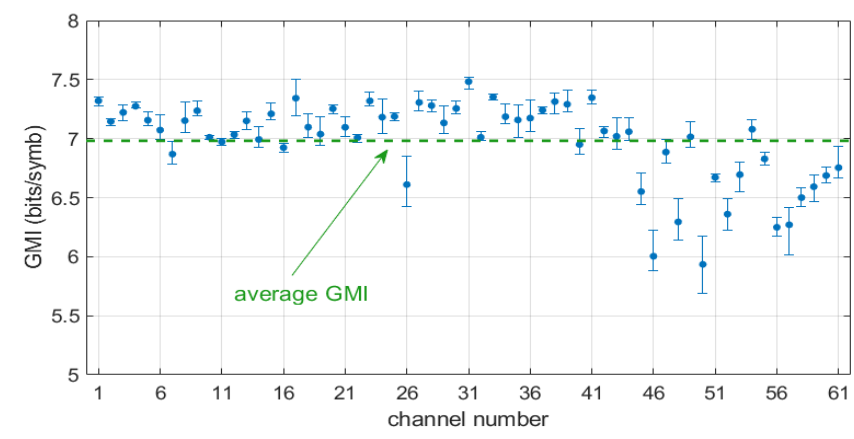

Fig. 10. PM-16QAM experiment. Total GMI (both polarizations) for all Cband channels at 26 recirculations (201 km through NANF). Six measurements per channel. Dots indicate the mean value, bars go from lowest to highest value. Higher channel index means higher channel frequency, from $191.9 \mathrm{THz}$ (channel \# 1) to $194.9 \mathrm{THz}$ (channel \# 61).

three impulse responses of the same set. This consistent normalization makes it possible to visually appreciate the different spread of the various impulse responses. In particular, for channel \#51 the plot clearly shows much more spread-out filter impulse responses, compatibly with the hypothesis of IMI being responsible for signal degradation.

Note in passing that IMI is theoretically unrecoverable by just equalization, since it is a non-unitary effect in our set-up: we only receive one mode and, in any case, modal attenuation is highly mode-dependent. Therefore, differently from (ideal) few-mode fibers with all-mode reception, singlemode reception with adaptive equalization cannot perfectly compensate for IMI. The equalizer helps, but cannot provide zero-penalty.

Also, in Fig. 9 we looked at the signal spectra before the LMS equalizer, measured at 80 recirculations, with VOA and with NANF, again for channel \# 31 and channel \# 51. The spectra with VOA are similar for the two channels and quite regular. Note that the enhancement of high-frequencies in the VOA spectra is due to the frequency response of the electronics. Instead, the jagged profile of the spectra with NANF once more appear to confirm the presence of substantial IMI-induced multi-path interference. In particular, the spectrum of channel \# 51 is quite degraded, providing further visual justification for its lower GMI.

One interesting remark is that the performance nonuniformity observed in Fig. 7 among channels was very repeatable over time. The six measurements for each channel were taken at long time intervals, days apart. We are however unsure what significance to attribute to this long-term repeatability.

Using simple analytical modeling (see Sect. VII-A for more details), we estimated that the degradation of performance between the case of VOA in the loop and NANF in the loop could be ascribed to a disturbance which was equivalent to a distributed IMI of about $-35.5 \mathrm{~dB} / \mathrm{km}$. Separate modeling of the NANF, after the experiment was completed, suggested that NANF 1 caused about $-50 \mathrm{~dB} / \mathrm{km}$ and NANF 2 about $-33 \mathrm{~dB} / \mathrm{km}$ of IMI and therefore that a line including only NANF 1 would in principle provide much improved performance.

NANF technology is still in its infancy and still in an exploratory phase, where different regions of their multidimensional design parameter space are being probed. This explains why NANF 1 and NANF 2 were fabricated with such different parameters and different resulting IMI. Note also that the strength of IMI is a parameter that is difficult to measure in the still typically short fiber lengths that can be produced. In particular, the strength of IMI of NANF 1 and NANF 2 was not known at the time when they were fabricated. The experiments reported here have provided useful feedback to this effect, which will be taken into account in future design refinements, and which will ensure that fibres more similar to NANF 1 (or actually better) will be used in any forthcoming experiment.

\section{PM-16QAM EXPERIMENT}

To test the possibility of transmitting a more complex constellation than PM-QPSK, we switched to PM-16QAM modulation, on the same experimental NANF set-up.

After preliminary characterization, the loop was set to 26 recirculations, or $201 \mathrm{~km}$ in NANF. The dots in Fig. 10 show the GMI (sum of both polarizations) of each C-band channel, averaged over 6 measurements. The corresponding bars range between the best and worst result for the same channel. The six measurements were taken on different days. Vertical bars range between min and max measured values. The mean GMI across all channels was 7 bits/symb.

This results represents the current record for PM-16QAM transmission over NANF (and on any HCF). It improves over the $124 \mathrm{~km}$ presented in [11]. It should also be noted that 
in [11] only $1 / 4$ of the channels were measured and that the back-to-back penalty was substantially lower $(0.9 \mathrm{~dB})$. This makes the result of this paper even more significant from the viewpoint of proving PM-16QAM long-distance transmission over NANF.

Here too, though, the presence of IMI was visible. Interestingly, as a general trend, there is a substantial correlation between channels being impaired by IMI in the PM-QPSK experiment and in the PM-16QAM experiment, as comparing Fig. 7 and Fig. 10 shows. In particular, the high index number channels are more impacted in both plots and the correspondence holds almost channel by channel.

However, the amount of degradation is substantially lower in the PM-16QAM experiment than in the PM-QPSK one. This is shown by the smaller ratio of worst to best channel GMIs, in the two experiments: 0.54 for PM-QPSK and only 0.8 for PM-16QAM. This is likely due to the lower number of recirculations in the PM-16QAM experiment vs. PMQPSK. The proportionality of the impairment to the number of recirculations appears to be a further confirmation of the IMI nature of the degradation.

\section{IMPACT OF L-BAND INTERFERERS}

The experimental set-up included both a C-band comb and an L-band comb. The overall optical spectrum is depicted in Fig. 3(a) and shows the $61 \mathrm{C}$-band channels together with the $61 \mathrm{~L}$-band ones. We remind the reader that all channels were ASE-emulated, except for the channel under test in the C-band. The schematic of the set-up, reported in Fig. 5, shows that the L-band channels were launched into the NANF together with the C-band channels, but were not recirculated. They were however co-propagating in the NANF and affected every recirculation of the $\mathrm{C}$-band comb. In practice, the $\mathrm{C}$-band channels interacted with the L-band interferers for the full NANF propagation length, $680 \mathrm{~km}$ in the case of PM-QPSK and $200 \mathrm{~km}$ in the case of PM-16QAM.

The reason why we performed this test was because we wanted to find out whether there was any cross-band interference effect in the NANF that could in principle prevent $\mathrm{C}+\mathrm{L}$ band transmission in the future. From a theoretical standpoint, based on the Kerr effect, the prediction was that $\mathrm{C}+\mathrm{L}$ interactions should be negligible at the power levels that are relevant for data transmission. On the other hand, NANF is a very new technology and we thought it would perhaps be risky to assume that we could rule out any non-linear crosstalk, without any experimental verification. Also, to the best of our knowledge, this was the first time such test has ever been run, at least for coherent WDM long-haul transmission.

As it turned out, we saw no effect of co-propagating the C-band with the L-band in the NANF. Specifically, we did not observe any significant change in GMI performance in the C-band channels, even at max reach, when the L-band interferers were turned on. Of course, ours tests represent only two individual data points (the PM-QPSK and PM-16QAM experiments) and all we can confidently state is that in these two experiments no effect was seen. It is perhaps not possible to draw general conclusion and we think that this aspect should be further explored by future experiments, perhaps operating at higher power levels. Nonetheless, the result is quite encouraging and hints at NANF being indeed capable of carrying very broad band signals without incurring detrimental cross-band effects, such as inter-channel stimulated Raman scattering (ISRS), which otherwise represent a substantial problem in solid-core fibers.

\section{COMments}

After conducting the transmission experiments (including the one presented in the next section) it was our intention to perform a deeper study of IMI, disassembling the NANF line into its NANF 1 and NANF 2 spools to test them separately and perhaps confirm the different IMI of the two spools. Unfortunately, immediately after OFC 2020, lockdown measures due to the Covid-19 pandemic were enforced at the laboratory location in Northern Italy. The experimental activity was stopped for many months. At present, the specific NANF line used for this experiment is not available and the possibility of bringing it back to the lab is made unlikely by new Covid19 related restrictions.

In any case, a key result emerging from the experiments reported in this paper is that reducing the level of distributed IMI in the fibres to the level of NANF 1 (or better) through refined designs has emerged as an essential requirement to enable further improvements in data transmission performance.

\section{A. Comparison with the previous experiment [11]}

The results shown in this paper substantially improve over the previous max-reach records in NANF [11]. With PMQPSK modulation, the reach of the center channel shown in this work is more than double that shown in [11] (note that a comparison of the WDM results is not possible since [11] only measured the center channel). Roughly similar relative improvements were found for PM-16QAM modulation as well. The question then may arise of why the present experiment outperforms [11] so substantially.

In essence, experiment [11] was a prior and first attempt, by the same groups authoring this paper, at long-haul WDM transmission over NANF. In retrospect, we realized that the experimental set-up [11] was not optimal for exploring the ultimate limitations to transmission performance caused by the NANF. By looking at Figs. 4 and 6 in [11] it appears that max-reach was induced by non-linearity. Such non-linearity was not due to the NANF, but to the $50 \mathrm{~km}$ SMF fiber that was present in the loop. In other words, the non-linearity of the SMF limited the capability of that experiment.

The set-up presented here was completely and carefully redesigned to avoid any such problems. The structure is much more complex, with 4 EDFAs in the loop rather than just 2. Ultra-low non-linearity PSCF was used rather than SMF to provide the indispensable signal buffer and loop stabilizing effect. The launch power into the PSCF was kept fixed at a level that made the non-linearity of the PSCF negligible.

The reach limitation in the current set-up is due to two main aspects: ASE noise accumulation and NANF propagation effects. The relative strength of the two can be tentatively 
estimated by comparing the results obtained when the NANF is in the loop and when the NANF is replaced by a VOA with same loss. Matching data with a simple model for the loop, which uses for the NANF the phenomenological crosstalk description [15], we tentatively estimate that the disturbance induced by the NANF is about three times larger at 80 recirculations than the accumulated ASE noise. The nonlinearity of the PSCF, estimated using the GN-model, is a further $13 \mathrm{~dB}$ below ASE and hence negligible.

While these are rough estimates, they suggest that the experimental set-up used in this experiment could indeed probe the transmission limitations due specifically to the NANF itself, and not be limited by other phenomena.

\section{CONCLUSION}

Nested Antiresonant Nodeless Fibers (NANFs) have been making steady progress over the last few years, to the point that state-of-the-art NANFs can now be used in long-haul WDM transmission experiments, a first for HCFs. In this paper we report on two recirculating loop transmission experiments over a hollow-core fiber of the Nested-Antiresonant Nodeless type (NANF).

We transmitted 61 channels in C-band at 32 GBaud into a recirculating loop, with either PM-QPSK or PM-16QAM modulation. In addition, $61 \mathrm{~L}$-band interferers co-propagated in the NANF at all times, though they were not recirculated in the loop, to check for the presence of possible crosstalk effects between $\mathrm{C}$ and L-band in the NANF.

The loop comprised the longest NANF transmission line yet constructed $(7.72 \mathrm{~km})$, as well as $55 \mathrm{~km}$ of PSCF, the latter needed to provide enough signal buffering and EDFA stabilization for the loop. The launch power into the PSCF was low enough to avoid generation of any significant nonlinear noise.

Using PM-QPSK modulation, we achieved a record $618 \mathrm{~km}$ transmission in NANF, at an overall average GMI of 3.44 bits/symb. This is $80 \%$ longer than the previous record (341 km) [11] where, however, only the center channel was measured. In the experiment described here, the center channel actually reached $772 \mathrm{~km}$.

Using PM-16QAM modulation, we achieved a record $201 \mathrm{~km}$ transmission, at an overall average GMI of 7 bits/symb. This is $60 \%$ longer than the previous record $(124 \mathrm{~km})$ [11] where, however, only $1 / 4$ of the WDM channels were measured.

We saw no adverse effect from the presence of L-band interferers in the NANF, at least at the power levels used in this experiment. This initial evidence bodes well for possible future NANF use in multi-band transmission systems. Our results also show that, besides loss, improvements are needed on the suppression of inter-modal interference, which seems possible with suitable design optimization.

If progress in NANF performance continues at the present rate, given its theoretical potential of achieving low loss and ultra-low non-linearity over ultra-wide bandwidths, it might become a promising alternative in the quest for next-generation higher-throughput fibers, ideally bringing about a many-fold increase in throughput per fiber [12].

\section{REFERENCES}

[1] R. F. Cregan, B. J. Mangan, J. C. Knight, T. A. Birks, P. St. J. Russell, P. J. Roberts and D. C. Allan, "Single-mode photonic band gap guidance of light in air,' Science, vol. 285, no. 5433, pp. 1537-1539, Sept. 1999.

[2] C. Peucheret et al., "10 Gbit/s transmission over airguiding photonic bandgap fiber at 1550 nm," Electron. Lett., vol. 41, no. 1, p. 27, 2005.

[3] F. Poletti, N. V. Wheeler, M. N. Petrovich, N. K. Baddela, E. Numkam Fokoua, J. R. Hayes, D. R. Gray, Z. Li, R. Slavik, and D. J. Richardson, "Towards high-capacity fibre-optic communications at the speed of light in vacuum," Nature Photonics, vol. 7, no. 4, pp. 279-284, 2013.

[4] V. A. J. M. Sleiffer, Y. Jung, N. K. Baddela, J. Surof, M. Kuschnerov, V. Veljanovski, J. R. Hayes, N. V. Wheeler, E. R. N. Fokoua, J. P. Wooler, D.R. Gray, N. H.-L. Long, F. R. Parmigiani, S.-U. Alam, M. N. Petrovich, F. Poletti, D. J. Richardson, and H. de Waardt, "High capacity modedivision multiplexed optical transmission in a novel 37-cell hollow-core photonic bandgap fiber," J. Lightw. Technol., vol. 32, no. 4, pp. 854-863, Feb. 2014.

[5] M. Kuschnerov et al., "Transmission of commercial low latency interfaces over hollow-core fiber," J. Lightw. Technol., vol. 34, no. 2, pp. 314-320, Jan. 2016.

[6] Y. Chen et al., "Multi-kilometer long, longitudinally uniform hollow core photonic bandgap fibers for broadband low latency data transmission," $J$. Lightw. Technol., vol. 34, no. 1, pp. 104-113, Jan. 2016.

[7] M.Kuschnerov et al., "Data transmission through up to $74.8 \mathrm{~km}$ of hollow core fiber with coherent and direct-detect transceivers," in Proc. European Conference on Optical Communications (ECOC), Valencia, Spain, paper Th1.2.4, Sept. 2015.

[8] F. Poletti, "Nested antiresonant nodeless hollow core fiber," Opt. Express, vol. 22, pp. 23807-23828, 2014.

[9] T. D. Bradley, G. T. Jasion, J. R. Hayes, Yong Chen, L. Hooper, H. Sakr, M. Alonso, A. Taranta, A. Saljoghei, H. C. Mulvad, M. Fake, I. A. Davidson, N. V. Wheeler, E. N. Fokoua, Wei Wang, S. Reza Sandoghchi, D. J. Richardson, F. Poletti, "Antiresonant Hollow Core Fibre with 0.65 $\mathrm{dB} / \mathrm{km}$ Attenuation across the $\mathrm{C}$ and L Telecommunication Bands," in Proc. European Conference on Optical Communications (ECOC), postdeadline paper PD.3.1, Dublin (IL), Sept. 2019.

[10] H. Sakr, T.D. Bradley, Y. Hong, G. T. Jasion, J. R. Hayes, H. Kim, I. A. Davidson, E. Numkam Fokoua, Y. Chen, K. R. H. Bottrill, N. Taengnoi, P. Petropoulos, D. J. Richardson, and F. Poletti. "Ultrawide Bandwidth Hollow Core Fiber for Interband Short Reach Data Transmission," in Proc. Optical Fiber Communications Conference (OFC), paper PDP Th4A.1, San Diego (CA), March 2019.

[11] A. Nespola et al., "Record PM-16QAM and PM-QPSK transmission distance (125 and $340 \mathrm{~km}$ ) over hollow-core-fiber," in Proc. European Conference on Optical Communications (ECOC), post-deadline paper PD.1.5, Dublin (IE), Sept. 2019.

[12] F. Poletti, P. Poggiolini, "Potential system impact of low-loss antiresonant hollow-core fibers," in Proc. European Conference on Optical Communications (ECOC), paper P51, Dublin (IE), Sept. 2019.

[13] G. T. Jasion, T. D. Bradley, K. Harrington, H. Sakr, Yong Chen, E. Numkam Fokoua1, I. A. Davidson, A. Taranta, J. R. Hayes, D. J. Richardson, F. Poletti "Hollow core NANF with $0.28 \mathrm{~dB} / \mathrm{km}$ attenuation in the C and L bands," in Proc. Optical Fiber Communications Conference (OFC), post-deadline paper Th4B.4, San Diego (CA), March 2020.

[14] A. Nespola, S. Straullu, T. D. Bradley, K. Harrington, H. Sakr, G. T. Jasion, E. Numkam Fokoua, Yongmin Jung, Yong Chen, J. R. Hayes, F. Forghieri, D. J. Richardson, F. Poletti, G. Bosco, P. Poggiolini, "Transmission of $61 \mathrm{C}$-band channels with L-band interferers over record 618 km of hollow-core-fiber," in Proc. Optical Fiber Communications Conference (OFC), post-deadline paper Th4B.5, San Diego (CA), March 2020.

[15] J. M. Gen, P. J. Winzer, 'A Universal Specification for Multicore Fiber Crosstalk,' IEEE Phot. Tech. Lett., vol. 31, no. 9, pp. 673-676, May 2019. 